\title{
A hidrocinesioterapia na funcionalidade, quedas e alterações musculoesqueléticas de idosas
}

\author{
Hydrokinesiotherapy on functionality, falls and musculoskeletal disorders in elderly women
}

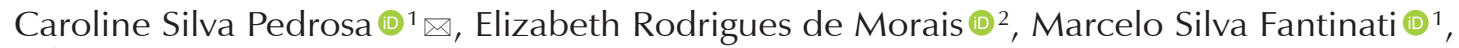 \\ Adriana Marcia Monteiro Fantinati (i) ${ }^{2}$ \\ ${ }^{1}$ Universidade Estadual de Goiás (UEG), Goiânia, GO, Brasil. \\ ${ }^{2}$ Universidade Estadual de Goiás (UEG) e Pontifícia Universidade Católica de Goiás (PUC-Goiás), Goiânia, GO, Brasil.
}

RESUMO

Objetivos: Avaliar os efeitos de um programa de hidrocinesioterapia na funcionalidade, risco de quedas e alterações musculoesqueléticas de idosas ativas participantes da Universidade Aberta à Terceira Idade da Pontifícia Universidade Católica de Goiás (UNATI PUC-GO). De forma secundária, objetivou-se correlacionar o perfil epidemiológico com a funcionalidade.

Materiais e Métodos: Estudo quase experimental, com uma amostra de 60 idosas, que frequentaram a oficina FISIOÁGUA, durante quatro meses, duas vezes por semana, por cinquenta minutos. Avaliou-se a funcionalidade pelo Short Physical Performance Battery (SPPB) e o risco de quedas pelo Quick Screen Clinical Falls Risk Assessment. A análise estatística foi realizada no SPSS utilizando-se o teste de Kolmogorov-Smirnov para determinar a normalidade dos dados, teste Wilcoxon para comparação e para correlação o índice de correlação de Pearson, com nível de significância de $p<0,05$.

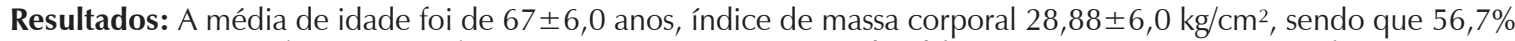
apresentavam Artrite/artrose e $40 \%$ Osteoporose. Foram encontradas diferenças estatisticamente significantes após a intervenção na funcionalidade $(p=0,0001)$ e risco de quedas $(p=0,0001)$. Ao se realizar uma análise de subgrupos, as idosas com Artrite/artrose e osteoporose após intervenção apresentaram melhora significativa na funcionalidade $(p=0,0001)$. Observou-se uma correlação estatisticamente significante, moderada e negativa entre a idade e a velocidade da marcha pré-intervenção $(r=-0,333, p=0,009)$. Após a intervenção $(r=-0,202, p=0,122)$ a correlação não foi significativa.

Conclusão: Observou-se que o programa de hidrocinesioterapia foi efetivo na melhora da funcionalidade, na diminuição do risco de quedas e alterações musculoesqueléticas de idosas ativas que apresentavam ou não artrite/ artrose e osteoporose.

Palavras-chave: idoso; hidroterapia; equilíbrio; quedas; funcionalidade.

\section{ABSTRACT}

\begin{abstract}
Objectives: To evaluate the effects of a hydrokinesiotherapy program on functionality, risk of falls and musculoskeletal changes of active elderly women who participate on the Universidade Aberta à Terceira Idade (Pontifícia Universidade Católica de Goiás - UNATI PUC-GO). Secondarily, we also aimed to correlate the epidemiological profile with the functionality.

Materials and Methods: Quasi-experimental study, with a sample of 60 elderly women who attended FISIOÁGUA workshop for four months, twice a week, by fifty minutes. The functionality was evaluated by the Short Physical Performance Battery (SPPB) and the risk of falls by the Quick Screen Clinical Falls Risk Assessment. The statistical analysis was performed using the SPSS. The KolmogorovSmirnov test was used to determinate the normality of the data, the Wilcoxon test for comparisons and to evaluate correlations the Pearson's correlation index was used, with a significance level of $p<0.05$.

Results: The mean age was $67 \pm 6.0$ years old, body mass index $28.88 \pm 6.0 \mathrm{~kg} / \mathrm{cm}^{2}$, of which $56.7 \%$ presented arthritis/arthrosis and $40 \%$ presented osteoporosis. Statistically significant differences were found after the intervention on functionality $(p=0.0001)$ and risk of falls $(p=0.0001)$. Through the subgroup analysis, after the intervention, the elderly with arthritis/arthrosis and osteoporosis presented significant improvement in the functionality $(p=0.0001)$. It was observed a statistically significant, moderate and negative correlation between age and pre-intervention gait speed ( $r=-0.333, p=0.009)$. After the intervention $(r=-0.202, p=0.122)$ the correlation was not significant.
\end{abstract}

Conclusion: It was observed that the hydrokinesiotherapy program was effective in improving the functionality, reducing the risk of falls and musculoskeletal alterations in active elderly women who presented or not arthritis and osteoporosis.

Keywords: elderly; hydrotherapy; balance; falls; functionality.

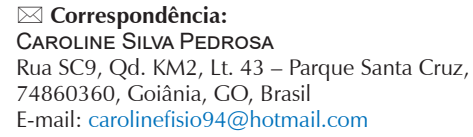




\section{INTRODUÇÃO}

A sociedade brasileira envelhece a passos $\operatorname{largos}^{1}$, as projeções atuais indicam um crescente aumento de idosos na população mundial. De acordo com a Organização Mundial de Saúde (OMS), em 2030 o Brasil terá a sexta população mundial em número de idosos ${ }^{1}$. Nesse contexto, esse fenômeno leva a uma série de implicações sociais, culturais e epidemiológicas ${ }^{2,3}$.

O processo de envelhecimento acarreta diminuição das funções fisiológicas e da resistência do corpo humano, tendo por consequência declínio da capacidade funcional. As modificações funcionais e estruturais nos sistemas do corpo humano decorrentes do envelhecimento aumentam o nível de dependência, comprometem o desempenho das atividades de vida diária (AVD's) e favorecem ainda o desenvolvimento de processos crônico degenerativos e comorbidades ${ }^{4,5}$.

As doenças relacionadas ao processo natural do envelhecimento que intensificam as deteriorações sensoriais e motoras, como a Artrite/Artrose e a Osteoporose podem por consequência levar a população idosa a ter déficits no controle postural ${ }^{6,7}$. Dentre os déficits funcionais no idoso as alterações do equilíbrio decorrentes do processo de envelhecimento estão associadas ao aumento do risco de quedas e apresentam como consequências o aumento de morbimortalidade, do risco de hospitalização, restrição de mobilidade e dificuldade em realizar as AVD ${ }^{\prime 4,8}$.

Os distúrbios relacionados ao equilíbrio vêm apresentando uma alta relevância clínica e tem se tornado um dos maiores problemas de saúde pública em idosos ${ }^{8}$. Portanto, nota-se a necessidade da investigação de recursos terapêuticos que proporcionem melhora da funcionalidade e do equilíbrio em idosos, visando um melhor direcionamento terapêutico e prevenção de complicações.

Uma das formas de minimizar os déficits na funcionalidade e o risco de quedas em idosos é a prática de atividades físicas, dentre elas a hidrocinesioterapia apresenta-se benéfica e efetiva para reduzir essas alterações advindas do processo de envelhecimento ${ }^{9}$. Os efeitos proporcionados pela água auxiliam na redução da dor, melhora da função física, força muscular, flexibilidade, da marcha e estabilização do tronco de forma segura ${ }^{10}$. Dessa forma, os exercícios terapêuticos na água têm sido importantes na prevenção, manutenção e melhora da funcionalidade e atuam de forma benéfica nas alterações do equilíbrio ${ }^{10-12}$.

Sendo assim, o presente estudo teve como objetivo avaliar o efeito da hidrocinesioterapia na funcionalidade, no risco de quedas e nas alterações musculoesqueléticas de idosas participantes da Universidade Aberta à Terceira Idade da
Pontifícia Universidade Católica de Goiás (UNATI PUC-GO), assim como verificar a correlação do perfil epidemiológico com a funcionalidade dessas idosas.

\section{MATERIAIS E MÉTODOS}

Trata-se de uma pesquisa de caráter analítico, quase experimental e de natureza epidemiológica. A amostra foi composta por 60 idosas ativas, participantes da UNATI PUC GO, que faziam parte exclusivamente da oficina FISIOÁGUA e não poderiam estar vinculadas a qualquer outra atividade física, sendo informadas desse compromisso no momento de assinarem o Termo de Consentimento Livre e Esclarecido.

Foi realizado um cálculo amostral no software Gpower 3.1.94, sendo considerado um alfa de 0,05, poder de 95\% e tamanho do efeito de 1 . A variável considerada para análise foi o SPPB total e a amostra necessária para o estudo foi de 13 pessoas.

Os critérios de inclusão foram: ser do sexo feminino, com idade igual ou superior a 60 anos, estar matriculada na UNATI PUC-GO, estar apta cognitivamente de acordo com o Mini-exame do Estado Mental ${ }^{13}$ com score mínimo de 17; ausência de doenças do tipo glaucoma e labirintite, ter 75\% de presença nos treinos cardiorrespiratórios na água por um período de quatro meses. Caso não possuíssem esse percentil de presença as participantes seguiriam normalmente o programa de exercícios, apenas não seriam incluídas no estudo. Participaram do estudo somente as idosas que assinaram o Termo de Consentimento Livre e Esclarecido, permitindo o início da coleta de dados.

Os critérios de exclusão foram: mulheres que não tenham passado pela triagem da UNATI PUC GO antes de participar do programa, que fossem participantes de outros estudos, tivessem mais de três faltas consecutivas e fossem portadoras de doenças neurológicas.

O sexo feminino foi escolhido pois a longevidade predomina nessa faixa etária. Ademais o sexo feminino é preditor de alterações do equilíbrio, devido ao declínio da capacidade funcional inerente ao processo de envelhecimento e aumento do número de condições crônicas mais evidentes nessa população se comparado ao sexo masculino ${ }^{14}$.

O estudo atende aos critérios éticos da Resolução 466/12 do Conselho Nacional de Saúde, foi aprovado pelo Comitê de Ética em Pesquisa da PUC GO (Protocolo no 968.807/2015) e todas as participantes assinaram o Termo de Consentimento Livre e Esclarecido (TCLE).

Os Instrumentos utilizados na pesquisa foram:

- Questionário epidemiológico: elaborado pelos próprios autores, utilizado para obtenção de dados sociodemográficos e sobre a saúde física das participantes, que apresentava questionamentos sobre a presença de algumas das seguintes 
doenças: cardiopatia, Hipertensão Arterial Sistêmica (HAS), Acidente Vascular Encefálico (AVE), diabetes mellitus (DM), câncer, reumatismo, pneumopatia, depressão, osteoporose, incontinência urinária e fecal e se faz uso de medicamentos.

- Short Physical Performance Battery (SPPB): utilizado para verificar os níveis de funcionalidade por meio de três testes: equilíbrio estático em pé; velocidade da marcha em passo usual, verificada em dois tempos em um percurso de ida e volta; e indiretamente a força muscular dos Membros Inferiores (MMII) com o teste de sentar e levantar sem auxílio dos Membros Superiores (MMSS). O escore de pontuação varia de zero (pior desempenho) a 12 pontos (melhor desempenho), sendo obtido pela soma da pontuação em cada teste ${ }^{15}$.

- Quick Screen Clinical Falls Risk Assessment: utilizado para avaliar o risco de quedas. Se o indivíduo apresentar nenhum ou um fator de risco, a probabilidade de cair é de $7 \%$, dois a três fatores de risco é de $13 \%$, quatro a cinco de $27 \%$ e seis ou mais $49 \%{ }^{16}$.

- Mini-Exame do Estado Mental (MEEM) ${ }^{13}$ : utilizado para avaliar o nível cognitivo dividido em sete categorias: orientação temporal e espacial, registro de três palavras, atenção e cálculo, recordação das três palavras, linguagem e capacidade construtiva visual. O escore varia de zero a trinta pontos, que indica a melhor capacidade cognitiva.

As avaliações foram em forma de entrevistas individuais realizadas em duas etapas: pré e pós-aplicação da hidrocinesioterapia. O estudo teve duração de quatro meses e foram esclarecidos os objetivos e a relevância da intervenção, além de outras dúvidas que surgiram sobre a pesquisa.

Em seguida, as idosas responderam às perguntas dos questionários epidemiológicos, saúde física, Quick Screen Clinical Falls Risk Assessement e MEEM aplicados por um dos pesquisadores previamente treinados. Após as avaliações, as participantes foram submetidas a avaliação da funcionalidade e do risco de quedas através do teste SPPB e Quick Screen Clinical Falls Risk Assessement, respectivamente, realizada de forma individual, em uma sala reservada na Clínica Escola Vida, tendo uma duração de aproximadamente 40 minutos.

Após a avaliação inicial as idosas iniciaram o programa de exercícios na água coordenado por uma Fisioterapeuta, duas vezes por semana, durante 50 minutos. No programa de hidrocinesioterapia foram aplicados exercícios sempre com o mesmo objetivo, respeitando uma sequência de: 10 minutos de aquecimento, 20 minutos de exercícios de força muscular e coordenação motora, associando a atividade de tronco, membros superiores e inferiores, e ao final, 10 minutos de exercícios respiratórios e alongamento global. Ao completar quatro meses no programa terapêutico as idosas foram reavaliadas na mesma metodologia supracitada.
Os dados coletados foram transcritos em uma planilha do Excel ${ }^{\circledR}$. A análise dos dados foi efetuada com o uso do programa Statistical Package for the Social Sciences (SPSS) versão 20.0. Foi realizada uma análise descritiva dos dados com cálculo de média e desvio padrão para as variáveis quantitativas e frequência e porcentagem para as variáveis qualitativas, para avaliar a normalidade utilizou-se o teste de Kolmogorov-Smirnov. Para comparação das variáveis antes e após a intervenção, utilizou-se o teste de Wilcoxon e para correlacionar o perfil epidemiológico com a funcionalidade, a correlação de Pearson. Considerou-se valores de r entre $0 \mathrm{e}$ 0,3 como correlação fraca, 0,31-0,69 correlação moderada e 0,70-1 correlação forte. Valores positivos foram considerados como correlação diretamente proporcional enquanto que valores negativos como correlação inversamente proporcional. O nível de significância adotado foi de $p<0,05$. Foi realizada uma análise por intenção de tratamento. Para verificar se os efeitos do programa se modificavam na presença de doenças musculoesqueléticas quanto à funcionalidade geral, foi realizada uma análise de subgrupos. Para comparação das variáveis antes e após a intervenção nos subgrupos do estudo também foi realizado o teste de Wilcoxon.

\section{RESULTADOS}

As características gerais das participantes do estudo estão demonstradas na Tabela 1. Observou-se que de forma geral as idosas do estudo apresentam sobrepeso e elevada circunferência abdominal. Dentre as participantes $40 \%$ eram casadas, $25 \%$ viúvas, $18,3 \%$ divorciadas e $16,7 \%$ eram solteiras, predominantemente com 1 o grau incompleto $40 \%$, $86,7 \%$ não eram etilistas e $93,3 \%$ não eram tabagistas. $\mathrm{Na}$ classificação ABEP, a classe C e D foram prevalentes com $33,3 \%$ e $48,3 \%$, respectivamente. No resultado do MEEM as participantes apresentaram uma média de 26 pontos, com a pontuação máxima de 30 e a mínima de 17 estando aptas cognitivamente.

Tabela 1. Características descritivas das idosas $(n=60)$.

\begin{tabular}{lcccc}
\hline & Média & DP & Máximo & Mínimo \\
\hline Idade (anos) & 67,0 & 6,0 & 82,0 & 60,0 \\
Massa corporal $(\mathrm{kg})$ & 68,0 & 12,9 & 109,0 & 44,0 \\
Altura $(\mathrm{m})$ & 1,53 & 0,06 & 1,75 & 1,31 \\
IMC $\left(\mathrm{kg} / \mathrm{cm}^{2}\right)$ & 28,8 & 5,0 & 47,1 & 20,8 \\
Circunferência abdominal $(\mathrm{cm})$ & 99,0 & 12,0 & 136,0 & 67,0 \\
\hline
\end{tabular}

n: Tamanho amostral; DP: Desvio padrão.

Na Tabela 2 estão representadas as condições de saúde física das idosas no qual predominou a artrite/artrose 56,7\%, seguido de osteoporose com $40 \%$. A comparação entre os 
momentos pré-intervenção e pós-intervenção das variáveis do SPPB estão demonstradas na Tabela 3. De acordo com os resultados apresentados, observaram-se diferenças estatisticamente significativas para as variáveis velocidades da marcha $(p=0,0001)$, sentar e levantar $(p=0,0001)$ e na pontuação geral do SPPB $(p=0,0001)$, porém no equilíbrio não houve diferença estatisticamente significativa $(p=0,317)$, em todos os casos o melhor desempenho foi visto após a hidrocinesioterapia.

As comparações intragrupos do SPPB estão demonstradas na Tabela 4. Observaram-se diferenças estatisticamente significantes no grupo com doenças musculoesqueléticas $(p=0,0001)$ e no grupo sem doenças musculoesqueléticas ( $p=0,0001)$ após o período de intervenção, observando-se uma melhora significativa em ambos os grupos.

Tabela 2. Descrição das condições de saúde física e do uso de medicamentos das idosas $(n=60)$.

\begin{tabular}{lcc}
\hline Doenças & Sim (\%) & Não (\%) \\
Doenças do coração, angina, Infarto & 11,7 & 88,3 \\
Hipertensão & 36,7 & 63,3 \\
Diabetes & 15,0 & 85,0 \\
Artrite/artrose & 56,7 & 43,3 \\
Depressão & 21,7 & 78,3 \\
Osteoporose & 40,0 & 60,0 \\
Medicamentos & 88,3 & 11,7 \\
\hline
\end{tabular}

n: Tamanho amostral; \%: Porcentagem.

Tabela 3. Análise comparativa dos valores do SPPB pré e póshidrocinesioterapia $(n=60)$.

\begin{tabular}{lccc}
\hline & \begin{tabular}{c} 
Pré-treinamento \\
\cline { 2 - 3 }
\end{tabular} & $\begin{array}{c}\text { Média (DP) } \\
\text { Equinamento }\end{array}$ & $\begin{array}{c}\text { Valor de } \\
\boldsymbol{p}^{*}\end{array}$ \\
Velíbrio & $3,93 \pm 0,36$ & $3,97 \pm 0,25$ & 0,317 \\
Sentar e levantar & $3,20 \pm 0,91$ & $3,87 \pm 0,38$ & $0,0001^{*}$ \\
SPPB Total & $10,15 \pm 1,48$ & $11,38 \pm 0,95$ & $0,0001^{*}$ \\
\hline
\end{tabular}

n: Tamanho amostral; DP: Desvio padrão; SPPB: Short Physical Performance Battery. * Teste de Wilcoxon, nível de significância de $p<0,05$.

Tabela 4. Comparação intragrupos do SPPB total pré e pós-treinamento na presença ou não das doenças musculoesqueléticas $(n=60)$.

\begin{tabular}{|c|c|c|c|c|}
\hline \multirow[b]{2}{*}{ SPPB } & \multicolumn{2}{|c|}{ GDM } & \multicolumn{2}{|c|}{ GSD } \\
\hline & Média (DP) & $\begin{array}{c}\text { Valor de } \\
\qquad p^{*}\end{array}$ & Média (DP) & $\begin{array}{l}\text { Valor de } \\
\qquad p^{*}\end{array}$ \\
\hline \multicolumn{5}{|c|}{ Osteoporose } \\
\hline Antes & $10,04 \pm 1,51$ & \multirow{2}{*}{$0,0001^{*}$} & $10,22 \pm 1,47$ & \multirow{2}{*}{$0,0001^{*}$} \\
\hline Depois & $11,2 \pm 0,83$ & & $11,50 \pm 1,02$ & \\
\hline \multicolumn{5}{|c|}{ Artrite/Artrose } \\
\hline Antes & $10,21 \pm 1,49$ & \multirow{2}{*}{$0,0001^{*}$} & $10,08 \pm 1,49$ & \multirow{2}{*}{$0,0001^{*}$} \\
\hline Depois & $11,44 \pm 0,70$ & & $11,31 \pm 1,22$ & \\
\hline
\end{tabular}

n: Tamanho amostral; DP: Desvio padrão; SPPB: Short Physical Performance Battery; GDM: Grupo com doenças musculoesqueléticas; GSD: Grupo sem doenças musculoesqueléticas. * Teste de Wilcoxon, nível de significância de $p<0,05$.
De acordo com os resultados apresentados na Tabela 5, sobre o risco de quedas, no pré-treinamento $50,9 \%$ da amostra apresentou um risco de queda equivalente a $7 \%$, classificação mínima, e no pós-treinamento, a porcentagem de participantes que apresentaram $7 \%$ de risco de quedas subiu para $80 \%$, apresentando melhora estatisticamente significativa $(p=0,0001)$.

Tabela 5. Resultados acerca do Quick Screen nas participantes $(n=60)$.

\begin{tabular}{|c|c|c|c|c|c|}
\hline \multirow{2}{*}{$\begin{array}{c}\text { Risco de Quedas } \\
\%\end{array}$} & \multicolumn{2}{|c|}{ Pré-treinamento } & \multicolumn{2}{|c|}{ Pós-treinamento } & \multirow{2}{*}{$\begin{array}{l}\text { Valor de } \\
\qquad p^{*}\end{array}$} \\
\hline & n & $\%$ & $\mathbf{n}$ & $\%$ & \\
\hline 7 & 29 & 50,9 & 48 & 80 & \multirow{4}{*}{$0,0001^{*}$} \\
\hline 13 & 23 & 40,4 & 8 & 13,3 & \\
\hline 27 & 5 & 8,8 & 3 & 5 & \\
\hline 49 & 3 & 5 & 1 & 1,7 & \\
\hline
\end{tabular}

n: Tamanho amostral; \%: Porcentagem.

* Teste de Wilcoxon, nível de significância de $p<0,05$.

$\mathrm{Na}$ análise de correlação entre o perfil epidemiológico e as variáveis do $S P P B$, foi verificada correlação estatisticamente significante, moderada e negativa entre a idade e a velocidade da marcha pré-intervenção $(r=-0,333$ e $p=0,009)$, sendo que após a intervenção essa mesma correlação não foi estatisticamente significativa $(r=-0,202$ e $p=0,122)$.

\section{DISCUSSÃO}

A proposta deste estudo foi avaliar a influência da hidrocinesioterapia na funcionalidade e nas alterações musculoesqueléticas das idosas ativas e os resultados apresentados sustentaram a hipótese e demonstraram que ela foi capaz de melhorar a funcionalidade avaliada por meio do teste SPPB das idosas que possuíam e não possuíam alterações musculoesqueléticas e ainda diminuir o risco de quedas.

Em relação ao IMC, a média da amostra apresentou-se com obesidade. As alterações do equilíbrio na população idosa, em indivíduos com sobrepeso e obesidade, acarretam limitações funcionais, que contribuem para aumentar o risco de quedas dessa população e pressupõem-se que a hidrocinesioterapia pode ter influenciado na diminuição ou manutenção do peso corporal, pois o exercício físico promove o aumento do gasto calórico atuando no controle da obesidade $\mathrm{e}^{17,18}$.

$\mathrm{Na}$ avaliação do desempenho físico funcional, os resultados demonstraram que a hidrocinesioterapia influenciou de forma significativa na melhora da velocidade de marcha, na força muscular dos membros inferiores, constatando a importância da intervenção proposta, tendo em vista que esses ganhos refletem na melhora da funcionalidade das idosas, uma vez que, uma pontuação 
menor representa maior incapacidade e aumento da mortalidade ${ }^{19-22}$.

O mesmo ocorreu nos estudos de Perracini \& Ramos ${ }^{14}$ e Fisken et al. ${ }^{23}$ nos quais a hidroterapia melhorou a velocidade de marcha e a força muscular de populações idosas. Entretanto, não houve uma padronização dos testes funcionais utilizados para avaliação dessas variáveis nessas idosas, o que dificulta a comparação quantitativa dos resultados encontrados.

Quanto à avaliação do equilíbrio estático, houve um aumento, porém não foi significativo e isso se justifica, porque as idosas do presente estudo foram consideradas ativas e pelo fato do teste utilizado avaliar apenas o equilíbrio estático, não conseguindo identificar maiores déficits no equilíbrio, uma vez que estudos realizados por outros autores $8,24,25,26$ identificaram uma melhora do equilíbrio utilizando outros métodos de avaliação que englobavam tanto o equilíbrio estático quanto o dinâmico.

As doenças musculoesqueléticas mais prevalentes foram a Artrite/Artrose e Osteoporose, similar aos achados de Melo et al. ${ }^{27}$ Observou-se ainda que todas as idosas possuíam alguma doença e de acordo com Santos et al. ${ }^{28}$ e Zaslavsky \& Gus ${ }^{29}$ são fatores predisponentes para alterações no equilíbrio e consequentemente favorecem o aumento do risco de quedas. Contudo, o presente estudo não observou diferenças estatisticamente significativas entre a funcionalidade das idosas quando foram divididas em grupos que apresentavam ou não essas doenças, mas isso se justifica pelo fato de serem idosas fisicamente ativas.

Na presente pesquisa, foi possível observar que tanto as idosas que possuíam ou não artrite/artrose e osteoporose apresentaram melhora significativa da funcionalidade após a realização da hidrocinesioterapia. Em um estudo com quinze idosos, com diagnóstico de Osteoartrite de joelho, foi avaliado o impacto da Fisioterapia aquática e da Fisioterapia terrestre na capacidade funcional e indicaram uma melhora significativa na capacidade funcional após intervenção no grupo que realizou exercícios aquáticos em comparação com exercícios no solo ${ }^{30}$.

Quanto à Osteoporose, os achados de Arnold et al. ${ }^{31}$ observaram melhora significativa no equilíbrio de idosas que realizaram exercícios aquáticos, quando comparado com exercícios no solo, já Devereux et al. ${ }^{32}$ identificaram melhora significativa do equilíbrio de idosas com diagnóstico de osteoporose que realizaram exercícios aquáticos em comparação com o grupo que não realizou nenhum exercício. Desse modo, o meio aquático é um ambiente de tratamento benéfico e seguro para pacientes com problemas musculoesqueléticos, que têm dificuldade em se exercitar no solo, por reduzir os riscos de quedas que podem causar incapacidades.

Em relação ao risco de quedas analisado pelo Quick Screen, os resultados evidenciaram que a hidrocinesioterapia foi capaz de diminuir significativamente o risco de quedas nas idosas, e consequentemente, atuar no aumento da funcionalidade, uma vez que a autonomia funcional tem uma forte relação com o risco de quedas em idosos ${ }^{33}$. Os estudos de Canderolo \& Caromano ${ }^{8}$ e Meereis et al. ${ }^{6}$ coincidiram com os achados do presente estudo e concluíram que a atividade realizada em meio aquático é eficiente para diminuir o risco de quedas de idosos.

Um dos preditores para a incapacidade funcional no idoso é a idade maior que 75 anos $^{34}$, que tem como consequência uma menor velocidade de marcha ${ }^{22,35}$. Os resultados encontrados pré-intervenção, indicaram uma correlação negativa entre a velocidade de marcha e a idade das idosas, este achado sustenta a hipótese de que o declínio da velocidade da marcha estaria relacionado à mudanças no sistema locomotor associadas ao envelhecimento ${ }^{18}$. Os resultados observados após a intervenção indicaram que a diminuição da velocidade de marcha não se mostrou muito expressiva nas idosas com idade mais avançada. Estes achados foram de particular relevância neste estudo, uma vez que evidenciam a importância de programas de exercícios terapêuticos destinados à população idosa, principalmente no ambiente aquático.

Este estudo contribui na prática clínica à medida que demonstra que um programa de hidrocinesioterapia realizado duas vezes por semana durante quatro meses é eficaz frente a não realização de exercício sobre a funcionalidade e o risco de quedas de idosas ativas. Algumas limitações ocorreram neste estudo, incluindo a ausência de grupo controle, carência de estudos utilizando o SPPB como instrumento para avaliar o desempenho físico funcional em idosos e o fato de não ter como garantir que as participantes não estavam realizando atividade física em outro local, apesar de ter sido esclarecido que não poderiam realizar. Logo, sugere-se para os próximos estudos, uma comparação com um grupo controle e comparação com outros métodos terapêuticos.

O presente estudo mostrou que a hidrocinesioterapia aumentou a funcionalidade, diminuiu o risco de quedas de mulheres idosas ativas e teve influência positiva na funcionalidade das que apresentavam disfunções musculoesqueléticas como a artrite/artrose e a osteoporose. Os resultados desse estudo ainda indicaram que quanto maior a idade menor a velocidade de marcha nas idosas avaliadas, porém após o programa de exercícios na água a idade não foi um fator que contribuiu significativamente para a diminuição da velocidade de marcha. 


\section{AGRADECIMENTOS}

À Universidade Aberta à Terceira Idade da Pontifícia Universidade Católica de Goiás (UNATI PUC-GO) pela concessão das idosas para participação da pesquisa.

\section{REFERÊNCIAS}

1. World Health Organization. Envelhecimento ativo: uma política de saúde. Brasília: Organização Pan-Americana da Saúde; 2005.

2. Nogueira SL, Ribeiro RCL, Rosado LEFPL, Franceschini SCC, Ribeiro AQ, Pereira ET. Fatores determinantes da capacidade funcional em idosos longevos. Rev Bras Fisioter. 2010;14(4):32229. http://dx.doi.org/10.1590/S1413-35552010005000019

3. Telarolli Júnior R, Loffredo LCM. Mortalidade de idosos em município do Sudeste brasileiro de 2006 a 2011. Ciênc Saúde Coletiva. 2014;19(3):975-84. http://dx.doi.org/10.1590/141381232014193.10482012

4. Ramos EC, Fonseca FF. Correlação entre fragilidade e risco de quedas em idosos da comunidade [trabalho de conclusão de curso]. Belo Horizonte: Universidade Federal de Minas Gerais; 2009. https://doi.org/10.17143/ciaed/xxiiciaed.2016.00299

5. Talley KM, Wyman JF, Gross CR, Lindquist RA, Gaugler JE. Change in balance confidence and its associations with increasing disability in older community-dwelling women at risk for falling. J Aging Health. 2014;26(4):616-36. https://doi. org/10.1177/0898264314526619

6. Toledo DR. Alterações sensoriais e motoras associadas ao envelhecimento e controle postural de idosos [dissertação]. Rio Claro: Universidade Estadual Paulista; 2008.

7. Xue QL. The frailty syndrome: definition and natural history. Clin Geriatr Med. 2011;27(1):1-15. https://doi.org/10.1016/j. cger.2010.08.009

8. Meereis ECW, Favretto C, Souza J, Marques CLS, Gonçalves MP, Mota CB. Análise do equilíbrio dinâmico de idosas institucionalizadas após hidrocinesioterapia. Rev Bras Geriatr Gerontol. 2013;16(1):41-7. http://dx.doi.org/10.1590/S180998232013000100005

9. Ruzene JRS, Navega MT. Avaliação do equilíbrio, mobilidade e flexibilidade em idosas ativas e sedentárias. Rev Bras Geriatr Gerontol. 2014;17(4):785-93. http://dx.doi.org/10.1590/18099823.2014.13105

10. Resende SM, Rassi CM, Viana FP. Efeitos da hidroterapia na recuperação do equilíbrio e prevenção de quedas em idosas. Rev Bras Fisioter. 2008;12(1):57-63. http://dx.doi.org/10.1590/S141335552008000100011

11. Cunha MF, Lazzareschi L, Gantus MC, Suman MR, Silva A, Parizi CC, Suarti AM, Iqueuti MM. A influência da fisioterapia na prevenção de quedas em idosos na comunidade: estudo comparativo. Motriz Rev Educ Fís. 2009;15(3):527-36.
12. Silva JMN, Barbosa MFS, Castro POCN, Noronha MM. Correlação entre o risco de queda e autonomia funcional em idosos institucionalizados. Rev Bras Geriatr Gerontol. 2013;16(2):337-46. http://dx.doi.org/10.1590/S1809-9823 2013000200013

13. Lourenço RA, Veras RP. Mini-exame do estado mental: características psicométricas em idosos ambulatoriais. Rev Saúde Pública. 2006;40(4):712-19. http://dx.doi.org/10.1590/S003489102006000500023

14. Perracini MR, Ramos LR. Fatores associados a quedas em uma coorte de idosos residentes na comunidade. Rev Saúde Pública. 2002;36(6):709-16. http://dx.doi.org/10.1590/S003489102002000700008

15. Nakano MM. Versão brasileira da Short Physical Performance Battery SPPB: adaptação cultural e estudo de confiabilidade [dissertação]. Campinas: Universidade Estadual de Campinas; 2007. https://doi.org/10.20396/ccfenf1201849

16. Tiedemann A, Lord SR, Sherrington C. The development and validation of a brief performance-based fall risk assessment tool for use in primary care. J Gerontol A Biol Sci Med Sci. 2010;65(8):896-903. https://doi.org/10.1093/gerona/glq067

17. World Health Organization. Obesity: preventing and managing the global epidemic. Geneva: WHO; 2000.

18. Aguiar JB, Paredes PFM, Gurgel LA. Análise da Efetividades de um programa sobre o equilíbrio, risco de quedas e o IMC de mulheres idosas. Rev Bras Ativ Fís Saúde. 2010;15(2): 115-9. https://doi. org/10.12820/rbafs.v.15n2p115-119

19. Costa AGS, Costa FBC, Oliveira ARS, Silva VM, Araujo TL. Ocorrência de quedas e índice de massa corporal em idosos. Rev Enferm. 2013;21(4):508-14.

20. Maciel ACC, Guerra RO. Prevalência e fatores associados ao déficit de equilíbrio em idosos. Rev Bras Ciên Mov. 2005;13(1):37-44. http://dx.doi.org/10.18511/rbcm.v13i1.610

21. Pavasini R, Guralnik J, Brown JC, di Bari M, Cesari M, Landi F, Vaes B, Legrand D, Verghese J, Wang C, Stenholm S, Ferrucci L, Lai JC, Bartes AA, Espaulella J, Ferrer M, Lim JY, Ensrud KE, Cawthon P, Turusheva A, Frolova E, Rolland Y, Lauwers V, Corsonello A, Kirk GD, Ferrari R, Volpato S, Campo G. Short physical performance battery and all-cause mortality: systematic review and metaanalysis. BMC Med. 2016;14(1):215. http://dx.doi.org/10.1186/ s12916-016-0763-7

22. Kirkwood RN, Gomes HA, Sampaio RF, Furtado SRC, Moreira BS. Spatiotemporal and variability gait data in community-dwelling elderly women from Brazil. Braz J Phys Ther. 2016;20(3):258-66. https://doi.org/10.1590/bjpt-rbf.2014.0157

23. Fisken AL, Waters DL, Hing WA, Steele M, Keogh JW. Comparative effects of 2 aqua exercise programs on physical function, balance, and perceived quality of life in older adults with osteoarthritis. J Geriatr Phys Ther. 2015;38(1):17-27. http://dx.doi. org/10.1519/JPT.0000000000000019 
24. Siqueira AF, Rebesco DB, Amaral FA, Maganhini CB, Dall Agnol SM, Furmann M, Mascarenhas LPG. Efeito de um programa de fisioterapia aquática no equilíbrio e capacidade funcional de idosos. Saude e Pesqui. 2017;10(2):331-8. https://doi. org/10.17765/1983-1870.2017v10n2p331-338

25. Franciulli PM, Souza GB, Albiach JF, Santos KCP, Barros LO, Santos NT, Souza FA, Bigongiari A, Barbanera M. Efetividade da hidroterapia e da cinesioterapia na reabilitação de idosos com histórico de quedas. Estud Interdiscipl Envelhec. 2015;20(3): 671-86.

26. Candeloro JM, Caromano FA. Efeito de um programa de hidroterapia na flexibilidade e na força muscular de idosas. Rev Bras Fisioter. 2007;11(4):303-9. http://dx.doi.org/10.1590/S141335552007000400010

27. Melo ACF, Nakatani AYK, Pereira LV, Menezes RL, Pagotto V. Prevalência de doenças musculoesqueléticas autorreferidas segundo variáveis demográficas e de saúde: estudo transversal de idosos de Goiânia/GO. Cad Saúde Colet. 2017;25(2): 138-43. http://dx.doi.org/10.1590/1414-462×2017000 10274

28. Santos FPV, Borges LL, Menezes RL. Correlação entre três instrumentos de avaliação para risco de quedas em idosos. Fisioter Mov. 2013;26(4):883-94. http://dx.doi.org/10.1590/ S0103-51502013000400017

29. Zaslavsky C, Gus I. Idoso: doença cardíaca e comorbidades. Arq Bras Cardiol. 2002;79(6):635-39. http://dx.doi.org/10.1590/ S0066-782X2002001500011

30. Barduzzi GO, Rocha Júnior PR, Souza Neto JC, Aveiro MC. Capacidade funcional de idosos com osteoartrite submetidos a fisioterapia aquática e terrestre. Fisioter Mov. 2013;26(2): 349-60. http://dx.doi.org/10.1590/S0103-515020130002 00012

31. Arnold CM, Busch AJ, Schachter CL, Harrison EL, Olszynski WP. A randomized clinical trial of aquatic versus land exercise to improve balance, function, and quality of life in older women with osteoporosis. Physiother Can. 2008;60(4):296-306. https://doi.org/10.3138/physio.60.4.296

32. Devereux K, Robertson D, Briffa NK. Effects of a water-based program on women 65 years and over: a randomised controlled trial. Aust J Physiother. 2015;51(2):102-8. https://doi.org/10. 1016/S0004-9514(05)70038-6

33. Silva JMN, Barbosa MFS, Castro POCN, Noronha MM. Correlação entre o risco de queda e autonomia funcional em idosos institucionalizados. Rev Bras Geriatr Gerontol. 2013;16(2):337-46. http://dx.doi.org/10.1590/S180998232013000200013

34. Pereira LC, Figueiredo MLF, Beleza CMF, Andrade EMLR, Silva MJ, Pereira AFM. Predictors for the functional incapacity of the elderly in primary health care. Rev Bras Enferm. 2017;70(1):106-12. http://dx.doi.org/10.1590/0034-7167-2016-0046
35. Pimenta FB, Pinho L, Silveira MF, Botelho ACC. Fatores associados a doenças crônicas em idosos atendidos pela estratégia de saúde da família. Ciênc Saúde Coletiva. 2015;20(8):2489-98. http:// dx.doi.org/10.1590/1413-81232015208.11742014

Autores:

Caroline Silva Pedrosa

Fisioterapeuta. Programa de Residência Multiprofissional, Universidade Estadual de Goiás (UEG).

Orcid: https://orcid.org/0000-0002-9765-8890

E-mail: carolinefisio94@hotmail.com

ELIZABETH RODRIGUES DE MORAIS

Fisioterapeuta. Doutora em Ciências da Saúde pela UFG. Professora da Universidade Estadual de Goiás (UEG) e da Pontifícia Universidade Católica de Goiás (PUC-Goiás).

Orcid: https://orcid.org/0000-0002-2944-4751

E-mail: elizabeth.r.morais@hotmail.com

MARCELO SILVA FANTINATI

Fisioterapeuta. Doutor em Patologia pela UFG. Professor do curso de Fisioterapia da Universidade Estadual de Goiás (UEG)

Orcid: https://orcid.org/0000-0002-1017-0014

E-mail: prof.fantinati@gmail.com

AdRIANA MARCIA MONTEIRO FANTINATI

Fisioterapeuta. Mestre em Ciência Ambientais e Saúde pela PUC-Goiás. Professora da Universidade Estadual de Goiás (UEG). e da Pontifícia Universidade Católica de Goiás (PUC-Goiás).

Orcid: https://orcid.org/0000-0001-7703-9390

E-mail: drifantinati@gmail.com 